\title{
BMJ Open Acceptability to patients of screening disposable transnasal endoscopy: qualitative interview analysis
}

\author{
John McGoran (D) , , Andrea Bennett, ${ }^{2}$ Joanne Cooper, ${ }^{2}$ John De Caestecker, ${ }^{1}$ \\ Laurence B Lovat, ${ }^{3}$ Neil Guha, ${ }^{2}$ Krish Ragunath, ${ }^{2}$ Sarmed S Sami ${ }^{3}$
}

To cite: McGoran J, Bennett A, Cooper J, et al. Acceptability to patients of screening disposable transnasal endoscopy: qualitative interview analysis. BMJ Open 2019;9:e030467. doi:10.1136/ bmjopen-2019-030467

- Prepublication history for this paper is available online. To view these files, please visit the journal online (http://dx.doi org/10.1136/bmjopen-2019030467).

Received 19 March 2019 Revised 27 October 2019 Accepted 01 November 2019

Check for updates

(c) Author(s) (or their employer(s)) 2019. Re-use permitted under CC BY-NC. No commercial re-use. See rights and permissions. Published by BMJ.

${ }^{1}$ Digestive Diseases Centre, University Hospitals of Leicester NHS Trust, Leicester, UK ${ }^{2}$ National Institute for Health Research (NIHR) Biomedical Research Center in Gastrointestinal and Liver Diseases, Nottingham University Hospitals NHS Trust, Nottingham, UK

${ }^{3}$ Division of Surgery and

Interventional Science,

University College London, London, UK

Correspondence to

Dr Sarmed S Sami;

s.sami@ucl.ac.uk

\section{ABSTRACT}

Objectives Screening in selected high risk populations for Barrett's oesophagus (B0) and oesophageal varices (OVs) has been proposed, but there are obstacles with conventional oesophagogastroduodenoscopy (C-OGD), including patient acceptability. Portable and disposable office-based transnasal endoscopy (TNE) is a feasible and accurate alternative to C-OGD that may have use in primary and secondary care. This article outlines a qualitative analysis of patient experiences of TNE and COGD in order to gain an insight into an acceptable delivery of an endoscopic screening service.

Design Purposeful sampling identified 23 participants who then underwent semi-structured interviews to determine their experiences of both procedures. Thematic analysis was conducted to derive meaning from their lived experiences.

Setting A secondary care endoscopy unit, clinic room and interview room.

Participants Patients referred for BO or OV surveillance and for endoscopy to investigate dyspepsia underwent unsedated TNE using the EG Scan II device followed by C-OGD with or without sedation (patient choice), as part of a clinical trial.

Results The themes that arose from our analysis were: inclusivity in one's own healthcare, comfort level and convenience, validity of the procedure and application to a screening population and a sense of altruism and reciprocity. Positive aspects of TNE included participant empowerment, reduced discomfort and avoidance of conscious sedation. Participants felt that if TNE screening was of proven efficacy it would be welcomed, though views on use in a community setting were mixed. Conclusions Most patients preferred TNE to unsedated C-OGD and the reasons they gave featured strongly in the emerging themes. Preferences between TNE and sedated C-OGD were more subtle, with equivalent comfort scores but merits and drawbacks of both being discussed. This information identifies opportunities and challenges in establishing an endoscopic screening service. Trialregistration number ISRCTNregistry identifier: 70595405; Pre-results.

\section{INTRODUCTION}

Portable and disposable office-based transnasal endoscopy (TNE) is a feasible and accurate alternative to conventional

\section{Strengths and limitations of this study}

The size of the original study, in which 100 patients from this centre alone allowed for a broad stated aim and collection of participants until data saturation was reached.

- Every participant had clinic-based transnasalendoscopy (TNE) before conventionaloesophagogastroduodenoscopy (C-OGD) so their experiences of the TNE would reflect that of a person undergoing screening more so than if $\mathrm{C}-\mathrm{OGD}$ was done first.

- The advantage in performing thematic analysis on the data gathered from interviews is that it allowed us to develop clear themes, which could allow transferability to other potential screening populations however this may be limited in non-research settings and other screening modalities.

- To maximise the credibility and dependability of the findings, reflexivity, in which the impact of the researcher and social environment on each other is acknowledged and managed, was employed at each stage. Care was taken not to manipulate participants into giving accounts that favoured or condemned either endoscopic test.

- An ordering effect cannot be quantified as all participants had TNE first and C-OGD second but we suggest that this order could have an impact on their perceptions of the endoscopic examinations.

oesophagogastroduodenoscopy (C-OGD) that may have use in primary and secondary care. $^{12}$ Several authorities have highlighted the need to identify alternative, minimallyinvasive screening tests for Barrett's oesophagus $(\mathrm{BO})$ and oesophageal varices (OV) which are precursor conditions for oesophageal adenocarcinoma (OAC) and variceal bleeding, respectively. ${ }^{3-6}$ The incidence and mortality from OAC have risen rapidly over the last few decades. ${ }^{7}$ This has prompted efforts to screen high risk patients who are predominantly white, male, age 55 or over, with chronic reflux symptoms for $\mathrm{BO} .{ }^{6}$ The prevalence in women is lower but exact proportions are unclear. Similarly, incidence 
and mortality from chronic liver disease is also rising, with one of the major contributors being variceal haemorrhage. ${ }^{8}$ It is recommended practice to screen for varices using endoscopy in all patients with cirrhosis but the costeffectiveness of this approach has been questioned. ${ }^{5}$

Currently, the standard of care for assessing $\mathrm{BO}$ and OVs is hospital-based C-OGD. This requires a fully equipped endoscopy department, complete with nursing support, recovery facilities, monitoring and decontamination rooms. ${ }^{9}$ Patients frequently require conscious sedation. ${ }^{10}$ C-OGD can be perceived to be an uncomfortable experience for the patient which may prompt those eligible for screening not to attend. ${ }^{11} 12$ These features of C-OGD fail to meet screening principles of cost-effectiveness and acceptability.

To overcome this, various alternative screening tools for high risk groups have been proposed and remain largely the preserve of research studies. Portable and disposable TNE as a method of screening for $\mathrm{BO}$ and OVs is one such tool that has been proposed as a solution. The suggested benefits of TNE over C-OGD include allowing the patient to speak during the examination, increased comfort levels and avoidance of sedation. It has been shown to be both accurate and cost-effective as a potential screening tool. ${ }^{121314}$ The EG Scan II system (Intromedic Ltd, Seoul, South Korea) is a transnasal endoscopic device with a disposable $6 \mathrm{~mm}$ diameter probe. It has an air insufflation channel but no biopsy or irrigation port and is designed to be a method of visualising various upper gastrointestinal
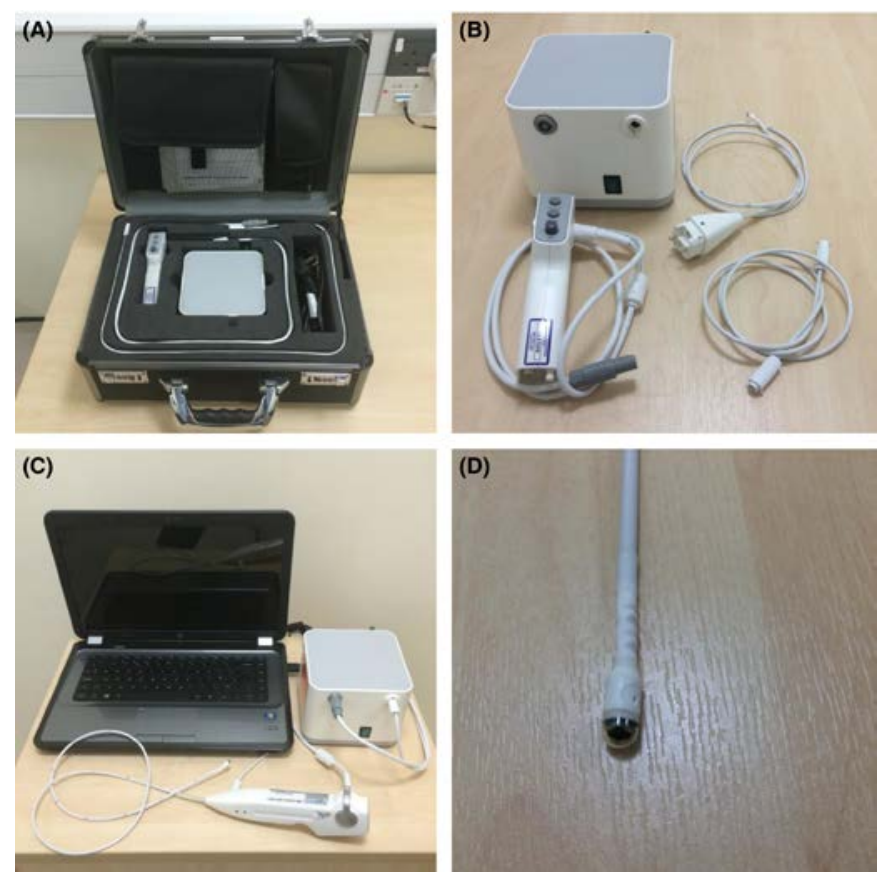

Figure 1 The EG Scan II system. (A) The portable case with four main parts; (B) the image processor (top left), disposable probe (top right), air tube (bottom right) and hand-held controller (bottom left); (C) the system connected and ready for use; (D) close view of the capsule probe tip. (Reproduced with permission from Sami, et al; Copyright, John Wiley and sons). conditions (figure 1). The apparatus has been designed for use in a consultation room, as a 'clinic-based' procedure, with potential for use in general practice.

The tolerability regarding comfort levels of TNE compared with that of C-OGD has been studied using visual scales and questionnaires in randomised trials, assessing attitudes towards and experience of sedated C-OGD and unsedated TNE that allow a simple measure of preference between the two. ${ }^{15}$ Acceptability is a deeper concept, that takes into consideration not only comfort scores but the global patient experience and willingness to undergo the intervention. It may act as an indicator of patient uptake and inform strategies for recruitment on a population level. To date, we have no knowledge of any qualitative studies that have considered the acceptability of TNE to patients who have undergone both it and C-OGD in a controlled environment. The lived experiences of those who undergo these procedures can inform preference and identify the challenges or opportunities for healthcare systems.

Tolerability and preference scores like the Visual Analogue Scale (VAS) and the Spielberger State-Trait Anxiety Inventory questionnaire are helpful ways of assessing the impressions of large populations of patients towards a healthcare experience. ${ }^{16}{ }^{17}$ They do not however permit reflection, nuance and richer insights into the experiences of individuals and therefore do not inform service development in the same way that qualitative methods do. A deeper analysis of these experiences could add to the clinical community's understanding of a patient's decision-making process and enhance human factors in screening or surveillance such as recruitment and retention.

The aims of this study were to explore the expectations and experiences of patients who underwent both TNE in an outpatient setting and C-OGD on the same day through qualitative analysis, and to determine the factors that impact on the acceptability of TNE as a potential screening and surveillance tool.

\section{METHODS}

\section{Study design and setting}

A qualitative methodology was employed, with the analysis of experiences and perceptions of both C-OGD and clinicbased TNE, using face-to-face semi-structured interviews. This builds on wider research examining the technical feasibility, quality, safety, acceptability (through scoring measures) and accuracy of clinic-based TNE using the EG Scan compared with C-OGD as the reference standard. ${ }^{1}$ A pictoral outline of this equipment is represented in figure 1 . Whereas the original research was conducted in three different centres, the collection of information with respect to this qualitative study was done at one centre (Nottingham, UK) with all patients having undergone both endoscopic procedures at that location.

Specific consent forms for these interviews were devised and confirmation of consent was obtained prior to each 
interview. Interview transcripts have been stored on an encrypted secure drive at the study site.

\section{Participants and recruitment}

Purposeful sampling took place, in which most of the individuals in one centre involved in the wider research project were identified as potential participants before being contacted by telephone within 4 weeks of the day of endoscopy. This is a common recruitment approach in qualitative research which contrasts with random sampling in quantitative research that seeks to reduce selection bias. Three patient groups were identified: those with histologically confirmed $\mathrm{BO}$, those warranting OV screening or surveillance and those with dyspepsia referred for diagnostic endoscopy. The experiences of all 47 patients were considered valid for an analysis of acceptability as they reflected a suitably healthy and communicative cohort, with three out of 50 ineligible because of ill health. Recruitment approaches for the quantitative and qualitative studies were done in parallel, with individuals approached for their consent to participate in an interview-based research study at the same time as for the quantitative study. They were then followed up within 4 weeks to ensure willingness to proceed with interview. Only the three individuals mentioned above withdrew consent or were withdrawn because of ill health. Those who could not tolerate both endoscopic examinations or who underwent variceal band ligation during C-OGD were excluded from recruitment to interview as their lived experiences were deemed to be considerably different from the rest. Sedated and unsedated patients were considered equally valid as this variation is reflected in clinical practice.

It was anticipated that a minimum of 20 interviews would have to be conducted to gather sufficient information. This was informed in part by existing literature. ${ }^{18}$ After 23 interviews were conducted, the researchers reviewed the merits of conducting further data collection as it would have required further appointments. After a preliminary period of data analysis it was proposed that saturation had been reached and no further participants would be required. The full thematic analysis of the existing interview transcripts supported this and it was agreed by the research team that further significant insights were unlikely to be gained from additional interviews.

\section{Data collection}

The interviews were conducted with interviewers guided by a topic schedule, that was agreed by consensus of the researchers. The scheduled questions related to all aspects of the endoscopic procedures and participants' views on the use of TNE in the community. Broad points included exploration of participants' prior understanding and expectations of undergoing both forms of endoscopy procedure, their experiences of the procedures and factors influencing this and their recommendations for future service provision, including any information or support needs. Box 1 outlines the interview schedule
Box 1 Schedule for use by research nurses conducting semi-structured interview

- Explore events that led to participant requiring endoscopy — ideas, concerns, expectations.

- Explore experiences of both tests - compare and contrast; short description.

- Ask about environment in which each test took place - description; feelings.

- Revisit visual analogue score to allow discussion over preferred test and explore reasons for this.

- Use participant's experience to ascertain their recommendations for delivery of both endoscopic tests.

- Explore thoughts over more widespread use of transnasal endoscopy for their condition - primary care.

further. The semi-structured nature of the interviews allowed the skilled researchers to explore the given answers with further probing and digression from the core topics. The topics and questions arose from the experiences of the researcher as clinicians in caring for patients undergoing endoscopy and knowledge of the processes of the original study where there was significant patient and public involvement. ${ }^{12}$ Both interviewers were female registered research nurses independent of the main study team and blinded to the patients' tolerability scores. The interviewers received site training to familiarise themselves with qualitative research and are subject to regular appraisal and research training, with oversight from a research team that includes an academic with a nursing background. The study was conducted and reported according to the Consolidated criteria for Reporting Qualitative research (COREQ) standards for qualitative research. $^{19}$

Interviews were recorded on an encrypted device and lasted between 20 and 35 min. Following this, recordings were transcribed to Microsoft Word documents undertaken by a professional transcriber who consciously checked their actions in retrospect and ensured that the transcripts were anonymised.

\section{Data analysis}

Transcribed interviews underwent thematic analysis by one researcher (JM) who was not involved in the study beforehand but fully briefed of the processes. Transcripts were read at least twice with broad notes being made of descriptions and sentiments from which possible recurrent themes could arise. ${ }^{20}$ The transcripts were then entered into NVivo V.12 (QSR International, Melbourne, Australia) software, which helped to identify the frequency of language used and its relation to other statements, to determine the relevance of the language used to the research aims. These patterns were again considered by the researcher and the themes were arrived at. Themes were again checked with the supporting text and interpretations drawn. 


\section{Rigour}

In order to ensure scientific rigour, checks were made at each stage of this study by primarily referring to the COREQ criteria, while taking care not to compromise the unique contribution that good quantitative research can bring to scientific progress. ${ }^{19} 21$ The research team also discussed the analysis frequently, using existing theoretical frameworks to ensure competent data collection and analysis. Our transcripts were analysed for deviant cases, which were noted to be equally valid to majority perspectives. As clinicians delivering endoscopic examination in daily practice, it was important for the team undertaking this qualitative analysis to exercise reflexivity. Consideration was made to the data collection, interpretation and arrival at themes through reflective discussion and selfanalysis. Specifically, awareness of the VAS for comfort from the original study was noted and care taken not to apply this to the qualitative analysis. With an acknowledgement of individual and collective biasses and motives, the researchers carefully tried to ensure that the themes arose from the participants' accounts alone. There may be a significant ordering effect resulting from TNE being performed before C-OGD in all cases. The quantitative study which analysed the diagnostic accuracy, safety, costeffectiveness and tolerability using VAS required this specific ordering. It does however represent a limitation in the case of the qualitative analysis. Involvement of the lead author (JM) who has no conflict of interest of note pertaining to device manufacturers, served to reduce the impact of apparent interpretative bias.

\section{Patient and public involvement}

The participants in this analysis were part of a larger study as noted earlier. Following TNE and C-OGD patients were asked to complete a questionnaire on their experiences of these tests. Analysis of the questionnaires and encounters between researchers and patients during this time strongly informed the preparation for semi-structured interviews. In addition, throughout this process the input of the patient and public involvement group at Nottingham Biomedical Research Centre was taken into consideration in terms of aims, design, patient consent and information leaflets. Details of the research study are to be made available to participants on request.

\section{RESULTS}

Twenty-three interviews took place, with participants having BO $(n=11)$, OVs $(n=8)$ or neither of these (those referred for investigation of dyspepsia) $(n=4$ (table 1$)$. Given the standard approach of the two endoscopic procedures, excluding those having had variceal band ligation, it was determined that all participants shared a similar lived experience and could all give accounts that can provide insights into the acceptability of TNE as a screening tool for either $\mathrm{BO}$ or OVs. For the purposes of the thematic analysis it was therefore not thought necessary to divide the groups according to their indication for endoscopy.
Table 1 Demographic and sedation details of 23 interview participants. Reflective of the general population for BO and cirrhotic liver disease, males represent the majority of participants. Sedation status for C-OGD was decided on by fully informed participants

\begin{tabular}{lc}
\hline \multicolumn{3}{l}{ Basic demographics of 23 interview participants } \\
\hline Males (n/23) & 17 \\
Sedation for C-OGD (n/23) & 13 \\
Median age (range) & $66(48-83)$ \\
Barrett's oesophagus (n male) & $11(9)$ \\
Liver disease warranting varices screening/ & $8(6)$ \\
surveillance ( $\mathrm{n}$ male) & \\
Dyspepsia (n male) & $4(2)$
\end{tabular}

BO, Barrett's oesophagus; C-OGD, conventional oesophagogastroduodenoscopy.

The majority $(17 / 23)$ of participants were men, which somewhat represents the gender balance in those having endoscopic examination for $\mathrm{BO}$ and OVs. The presence of sedation for C-OGD was a factor in participants' lived experiences and this was taken into account during interview of each individual. Recall of events differed between participants because of sedation however the absence of perceived discomfort is as important a feature of acceptability as conscious and remembered tolerability, when considering attitudes towards this test. The inconvenient aspects of intravenous sedation such as increased length of clinical observation and a 'hangover effect' were also discussed.

Four themes were identified after thematic analysis. The four themes were: (i) inclusivity in one's own healthcare, (ii) comfort level and convenience, (iii) validity of the procedure and application to a screening population, (iv) a sense of altruism and reciprocity. The evidence supporting these four themes is outlined below, illustrated with excerpts from transcripts.

\section{Inclusivity in one's own healthcare}

On attending for the dual endoscopic tests as part of a research study, most participants described feeling wellinformed of the procedures and involved in the decisionmaking process. Two people expressed initial wariness of TNE as they did not know what to expect but felt reassured after a fuller explanation. Every participant acknowledged that they had consented with appropriate knowledge of each procedure. TNE was described as 'empowering', 'fascinating' and a participant stated that they would 'definitely' have it done again. Specific comments regarding TNE's merits included that they could speak with the operator, sit up in a chair and view their endoscopic images if they wished - I could watch it on the monitor' (male, 70, dyspepsia).

By contrast, the 'impersonal' C-OGD made participants feel 'trapped' and 'claustrophobic' or like in 'a production line'. Comments were highly suggestive 
of a disinclination to undergo C-OGD. Unfavourable comments about C-OGD were underlined by participants' responses to queries over their involvement in the decision making process - 'I could have said no. But I came looking for help' (male, 70, dyspepsia), 'as a patient, I have the ultimate say' (male, 66, liver disease). The existence of established guidelines, best practice and trust in the decisionmaking of medical professionals prompted attendance for endoscopic tests, with one participant summarising the feelings of many others by saying 'If you've got to have a test, you've got to have a test'. Another participant said of C-OGD, 'I know it's part of the care, that you've got to have it done' (female, 83, Barrett's). Participants used their knowledge of their chronic conditions and their symptoms as accompanying motives for having endoscopic tests.

\section{Comfort level and convenience}

As part of the original study, every participant completed visual analogue scales of their experiences of each endoscopic procedure. The interviewers had access to the VAS for each participants and reminded them of the score out of 10 they gave, before exploring the reason for this and any change in attitude made in retrospect. This part of the data collection reaffirmed that the majority preferred TNE to unsedated C-OGD. Among those having sedation for C-OGD, the preference for TNE remained but this was less strong. Of the 13 who chose sedation, all but one had experienced a C-OGD in the past and some stated this choice was informed by past experiences of undergoing endoscopy without. All TNEs were performed without sedation, which was reported as acceptable by all participants, who described minor nasal discomfort as the predominant uncomfortable element. They had insight that having an unsedated procedure would mean 'you can go straight home' (female, 83, Barrett's) afterwards, making it more time-efficient and convenient. For those who had unsedated C-OGD, excessive gagging featured almost universally as well as a measure of distress that caused one patient to cry and another to state 'I wouldn't recommend that to anyone' (male, 65, dyspepsia). Gagging was less of an issue for TNE as described by the individual who reported, 'not so much with the nasal one but with the, with the oral one, it was very bad gagging reflex' (male, 75, dyspepsia). Beyond this theme, patient experiences and accounts did not appear to be affected by their sedation status.

\section{Validity of the procedure and application to a screening population}

The EG Scan has been shown to have accuracy in the detection of $\mathrm{BO}$ and medium/large OVs. ${ }^{12}$ Participants were informed of the outcome of their own endoscopic examinations but were unaware of the study findings that TNE and C-OGD results were in concordance. Participants were however encouraged to use their lived experience to give an account of how important test accuracy would be to them and to others. The premise was that of TNE being extended to screening individuals in a community setting.
For those who had a history of undergoing C-OGDs for BO and OVs, the potential accuracy of TNE was important. They described C-OGD as a 'necessary evil' but an informative test for their condition which they have little choice but to undergo given the reasons above. Having been briefed on the devices, one participant recognised a possible limitation of the EG Scan probe - 'The nasal one, you can't actually take biopsies from, you can't use that to take samples' (male, 72, Barrett's). While most acknowledged TNE as more comfortable, they suggested that it would only be an acceptable alternative if its ability to detect abnormalities matched that of C-OGD. Based on his experience, ('the day I had them both done, I think the nasal one, I think it missed something out' (male, 58, Barrett's)) one participant declared, 'I do believe the gastroscopy one is more thorough' (male, 71, liver disease). Alongside this, the participants emphasised the requirement for adequately trained endoscopists to perform TNE and some expressed reservations over their general practitioners (GPs) taking up the role - 'As long as you had proficient GPs doing it', 'As long as he (GP) can do it...properly' (male, 68, Barrett's). For this reason, opinion was divided on the location of the test, with some preferring the specialist nature of secondary care and others espousing the greater convenience and comfort of having TNE in the community.

\section{Sense of altruism and reciprocity}

Participants used their experiences as patients and as study participants to deliver unique insights into how endoscopic screening would be received by the target populations. For many patients, their positive experiences of healthcare informed their decision-making in becoming a research participant. 'I said yes because I was most grateful for what the hospital had done for me previously' (female, 59, Barrett's), 'I ought to try and put something back in' (female, 60, liver disease) and 'T'm always ready to help in the progress of science' (male, 65, dyspepsia) were some statements that clearly showed the willingness of participants to use their experiences for the betterment of others.

The involvement of those who have undergone the procedure is an important factor in establishing a screening programme. Participants suggested that prospective patients would be reassured by engaging with someone who had already undergone the procedure, reflecting the place of social constructionism in society's view towards healthcare screening in an overt fashion. ${ }^{22}$

\section{DISCUSSION}

This research represents the first time that a qualitative study has been conducted into the experiences of patients undergoing transnasal endoscopy for screening of gastrointestinal conditions. The aim of this research was not to contrast one endoscopic mode with the other, as each has its own applicability in clinical practice, but to consider the acceptability of TNE in screening of high risk individuals. The additional information derived from a qualitative as compared with a quantitative means of assessing 
acceptability (eg, VAS or structured questionnaire) allows for deeper interpretation of a patient's perspective. The themes identified in this study support the view that TNE could be acceptable to a screening population, thus fulfilling an important principle of a viable screening programme. ${ }^{23}$

The community of inquiry theory is a concept that unites the themes identified in this study. ${ }^{24}$ The background is that TNE is shown to be accurate and safe in the delivery of $\mathrm{BO}$ and $\mathrm{OV}$ assessment but the problem remains that not enough is deeply understood about its acceptability to a population. To explore the challenges and advantages offered by TNE in the screening of BO and OVs, male and female participants of different ages and for different reasons underwent the same procedure. Their individual perspectives were all considered and conclusions that reflect this community's account were arrived at, which will hopefully contribute to improved delivery of patient care. For the purposes of this study all of the accounts were communicated through the researchers but they are reflective of the conversations that may exist in the greater population.

To the participants, the notion of an accurate test that was superior in comfort and convenience was appealing. They could see the benefits that this could bring a wider population and were pleased to be part of a community that could enhance other patients' experiences. The greater mutualism and control that TNE seems to bring in contrast to C-OGD made it more appealing, which could be important in achieving adequate uptake of in a screening population. It must of course be noted that the theme of 'inclusivity in one's own healthcare' that arose from the interviews is within a study setting of participants who volunteered to undergo TNE, in contrast with a screening population who may feel less able to decline. The evidence from the accounts nevertheless supports the impression that TNE can enhance a feeling of involvement, which is an important factor in uptake and concordance. In a public health setting, inclusivity and a sense of community of which altruism is a component, are essential factors affecting patient involvement. Questions for the use of TNE as a screening tool remain. The inability of the EG Scan system to allow biopsies was one recurring concern for participants, and nasal discomfort is a significant drawback. There is evidence for higher comfort levels during endoscopy when thinner endoscopes $(6 \mathrm{~mm}$ or less in diameter) are used, whether transnasally or transorally and a different device might be used to achieve this. ${ }^{25}$

Contradicting views emergent from the data also provide important insights. The minority of participants who did not wish to view endoscopic images, those who would prefer C-OGD as a screening tool because of its established nature and individuals who would prefer to attend hospital for TNE rather than the community, all had valid reasons that warrant consideration. The participants realised that, as individuals who had pre-existing conditions or symptoms requiring OGD examination, they differed from a screening population, making direct comparisons impossible. Some of the reasons given for preferring aspects of C-OGD over TNE are indeed based in existing practices for example, oesophageal biopsies in BO using C-OGD.

Qualitative research of patients undergoing C-OGD alone for $\mathrm{BO}$ reflect themes of comfort and control being important to them, which aligns well with our findings. ${ }^{26}$ Additionally, qualitative analysis of interviews has been conducted to gain an insight into factors affecting adherence to endoscopic screening, which would be a major factor in establishing an effective service. ${ }^{27}$ A clearer view of the population's interpretation of such an intervention may enhance the confidence of healthcare providers to establish a screening programme with TNE at its core.

\section{CONCLUSIONS}

The information collected in this patient-centred qualitative study has identified four main themes underlying the expectations and experiences of those undergoing TNE and C-OGD. They are inclusivity in one's own healthcare, comfort level and convenience, validity of the procedure and application to a screening population and a sense of altruism and reciprocity. We believe that this analysis is a credible reflection of the experiences of the patients involved and that it provides insights into the opportunities and challenges for interventions offered to patients selected as 'high risk' and therefore considered for screening. These insights could not have arisen from quantitative analyses of acceptability and highlight the strengths of qualitative methods to optimise clinical care. The themes align with the original study's VAS outcomes in suggesting that unsedated TNE is potentially acceptable and may reflect the experiences of high risk screening populations within the wider community.

Twitter Joanne Cooper @drjo_cooper

Contributors JM: contributed to analysis, interpretation of data and drafted the manuscript. $A B$ : contributed to the acquisition of data and critical revision of the manuscript for important intellectual content. JC: contributed to acquisition of data, interpretation of data and critical revision of the manuscript for important intellectual content. JDC: contributed to the study supervision, interpretation of data and critical revision of the manuscript for important intellectual content. LBL: contributed to the study supervision, interpretation of data and critical revision of the manuscript for important intellectual content. NG: contributed to the acquisition of data, study supervision, interpretation of data and critical revision of the manuscript for important intellectual content. KR: contributed to the conception, design and supervision of the study, acquisition of data, study supervision, interpretation of data and critical revision of the manuscript for important intellectual content. SSS: contributed to the conception, design and supervision of the study, acquisition of data, analysis, interpretation of data and critical revision of the manuscript for important intellectual content.

Funding SSS was funded by an Olympus-Core National Endoscopy Research Fellowship grant, Core Charity, United Kingdom (grant number: RB4803). This study was part funded by a grant from Intromedic Ltd, Seoul, South Korea.

Competing interests KR is a research grant recipient from Intromedic Ltd, Seoul, South Korea.

Patient consent for publication Not required.

Ethics approval The East Midlands division of the Research Ethics Committee granted approval for the study (REC reference 12/EM/0100). 
Provenance and peer review Not commissioned; externally peer reviewed.

Data availability statement Data are available upon reasonable request.

Open access This is an open access article distributed in accordance with the Creative Commons Attribution Non Commercial (CC BY-NC 4.0) license, which permits others to distribute, remix, adapt, build upon this work non-commercially, and license their derivative works on different terms, provided the original work is properly cited, appropriate credit is given, any changes made indicated, and the use is non-commercial. See: http://creativecommons.org/licenses/by-nc/4.0/.

ORCID iD

John McGoran http://orcid.org/0000-0002-9514-5290

\section{REFERENCES}

1 Sami SS, lyer PG, Pophali P, et al. Acceptability, accuracy, and safety of disposable transnasal capsule endoscopy for Barrett's esophagus screening. Clin Gastroenterol Hepatol 2019;17.

2 Sami SS, Ragunath K, Wilkes EA, et al. The detection of oesophageal varices using a novel, disposable, probe-based transnasal endoscope: a prospective diagnostic pilot study. Liver Int 2016;36:1639-48.

3 Shaheen NJ, Falk GW, lyer PG. ACG Clinical Guideline : Diagnosis and Management of Barrett' s Esophagus. Am J Gastroenterol 2015:1-21.

4 Garcia-Tsao G, Abraldes JG, Berzigotti A, et al. Portal hypertensive bleeding in cirrhosis: risk stratification, diagnosis, and management: 2016 practice guidance by the American association for the study of liver diseases. Hepatology 2017;65:310-35.

5 Tripathi D, Stanley AJ, Hayes PC, et al. U.K. guidelines on the management of variceal haemorrhage in cirrhotic patients. Gut 2015;64:1680-704.

6 Fitzgerald RC, di Pietro M, Ragunath K, et al. British Society of gastroenterology guidelines on the diagnosis and management of Barrett's oesophagus. Gut 2014;63:7-42.

7 Hayeck TJ, Kong CY, Spechler SJ, et al. The prevalence of Barrett's esophagus in the US: estimates from a simulation model confirmed by SEER data. Dis Esophagus 2010;23:451-7.

8 Thomopoulos K, Theocharis G, Mimidis K, et al. Improved survival of patients presenting with acute variceal bleeding. prognostic indicators of short- and long-term mortality. Dig Liver Dis 2006;38:899-904.

9 Dunkley I, Griffiths H, Follows R, et al. Uk consensus on nonmedical staffing required to deliver safe, quality-assured care for adult patients undergoing gastrointestinal endoscopy. Frontline Gastroenterol 2019;10:24-34.

10 Cohen LB, Delegge MH, Aisenberg J, et al. AGA Institute review of endoscopic sedation. Gastroenterology 2007;133:675-701.
11 Kruijshaar ME, Kerkhof M, Siersema PD, et al. The burden of upper gastrointestinal endoscopy in patients with Barrett's esophagus. Endoscopy 2006;38:873-8.

12 Beg S, Ragunath K, Wyman A, et al. Quality Standards in upper gastrointestinal endoscopy: a position statement of the British Society of gastroenterology (BSG) and association of upper gastrointestinal surgeons of great britain and ireland (AUGIS). Gut 2017;66:1886-99.

13 Aedo MR, Zavala-González Miguel Á, Meixueiro-Daza A, et al. Accuracy of transnasal endoscopy with a disposable esophagoscope compared to conventional endoscopy. World J Gastrointest Endosc 2014;6:128-36.

14 Honing J, Kievit W, Bookelaar J, et al. Endosheath ultrathin transnasal endoscopy is a cost-effective method for screening for Barrett's esophagus in patients with GERD symptoms. Gastrointest Endosc 2019;89:712-722.e3.

15 Blevins $\mathrm{CH}$, Egginton JS, Shah ND, et al. Comparative assessment of patient preferences and tolerability in Barrett esophagus screening: results from a randomized trial. J Clin Gastroenterol 2018;52:880884.

16 Likert R. A technique for the measurement of attitudes. Arch Psychol 1932;22.

17 Marteau TM, Bekker H. The development of a six-item short-form of the state scale of the Spielberger State-Trait anxiety inventory (STAl). Br J Clin Psychol 1992;31:301-6.

18 Guest G, Bunce A, Johnson L. How many interviews are enough?: an experiment with data saturation and variability. Field methods 2006;18:59-82.

19 Tong A, Sainsbury P, Craig J. Consolidated criteria for reporting qualitative research (COREQ): a 32-item checklist for interviews and focus groups. Int J Qual Health Care 2007;19:349-57.

20 Braun V, Clarke V, Terry G. Thematic analysis. Qual Res Clin Heal Psychol 2014;24:95-114.

21 Barbour RS. Checklists for improving rigour in qualitative research: a case of the tail wagging the dog? BMJ 2001;322:1115-7.

22 Calvo A, Brown KM, McDermott RJ, et al. Social construction of cervical cancer screening among Panamanian women. Am J Health Educ 2012;43:153-63.

23 Wilson JMG, Jungner G. Principles and practice of screening for disease. World Health Organization, 1968.

24 Garrison DR, Anderson T. E-Learning in the 21stCentury: a framework for research and practice. London: Routledge/Falmer, 2003.

25 Lin L-F, Ma K-Z, Tu H-L. A prospective randomized study comparing transnasal and peroral 5-mm ultrathin endoscopy. J Formos Med Assoc 2014;113:371-6.

26 Arney J, Hinojosa-Lindsey M, Street RL, et al. Patient experiences with surveillance endoscopy: a qualitative study. Dig Dis Sci 2014;59:1378-85.

27 Sly JR, Edwards T, Shelton RC, et al. Identifying barriers to colonoscopy screening for nonadherent African American participants in a patient navigation intervention. Health Educ Behav 2013;40:449-57. 